\title{
Utilisation des plans d'expériences pour évaluer la robustesse d'une méthode d'analyse quantitative par Chromatographie en Phase Liquide
}

\author{
J. Vial et A. Jardy \\ Laboratoire environnement et chimie analytique (associé au CNRS) Ecole supérieure de physique \\ et de chimie de Paris, 10, rue Vauquelin, 75231 Paris Cedex 05, France
}

\begin{abstract}
The ruggedness of an analytical method is defined by its capacity to remain unaffected (e.g. to maintain its performances) when small changes in experimental conditions happen. It can be studied by applying changes within the same order of magnitude of those which could occur when running routinely the method. In the case of a L.C. method, given the high number of factors (temperature, flow rate, mobile phase composition, detection wavelength, injected sample quantity...) susceptible to vary and the possible interactions between them, varying each factor independently would be awkward. The experimental design method enables to model the effects and interactions of a high number of factors varying simultaneously with a limited number of runs. After having estimated the order of magnitude of the potential changes in the factors level, a central composite design, allowing independent estimations of model coefficients, must be carried out around the working point defined during the method optimisation. Such a design allows the use of a second order model and gives the possibility to have an extremum inside the experimental domain. So it is possible to predict the consequences of small variations in operating conditions on the responses. A global function is proposed to account for ruggedness in terms of capacity for the method to deliver steady and reproducible assays for a product, which represents the aim of quantitative analysis. A statistical treatment of the design results reveals that the percentage of acetonitrile in the mobile phase, the column temperature and the $\mathrm{pH}$ of the mobile phase are the factors that have a significant influence on the method ruggedness and are critical. Hence their identification leads to recommendations in the operating protocol ; the ruggedness of the analytical method will be considered as maintained only when the tolerances defined for those parameters judged critical are respected.
\end{abstract}

Key words. Ruggedness - HPLC - design of experiments - quality control - method validation.

\section{Introduction}

Plusieurs éléments entrent dans la validation d'une méthode d'analyse par chromatographie en phase liquide (CPL); il faut à ce stade vérifier que le cahier des charges est correctement rempli (séparation des différents composés satisfaisante, temps d'analyse non rédhibitoire, limites de détection et de quantification compatibles avec les niveaux attendus, linéarité, répétabilité etc...). En général, la dernière partie du processus de validation est consacrée à la « robustesse » de la méthode [1]. Mais comme cette notion est encore à l'heure actuelle assez mal appréhendée, elle n'est souvent abordée que de façon trop partielle.

La robustesse d'une méthode d'analyse est sa capacité à rester stable (maintenir ses performances) face à de faibles variations des conditions expérimentales, variations de l'ordre de celles qui pourraient se produire lors de la mise en application de la méthode. C'est une caractéristique essentielle en contrôle qualité. L'exemple choisi ici porte sur la détermination du titre d'un produit et de ses substances apparentées par CPL Le produit choisi pour mener l'étude est la spiramycine (Rhône-Poulenc Rorer). Son analyse chromatographique est délicate étant donné que les principes actifs et les substances apparentées sont difficiles à séparer. De plus, comme on le verra, la méthode chromatographique utilisée est très sensible aux conditions de l'analyse. Toutes ces caractéristiques font que ce problème se prête très bien à la mise au point d'une méthodologie d'étude de robustesse en analyse quantitative en utilisant la technique des plans d'expériences [2-5]. L'objectif de l'étude n'est pas de reprendre l'optimisation de la méthode, mais d'examiner l'incidence de faibles variations fortuites des facteurs autour de leurs valeurs de consigne sur ses performances.

Après avoir brièvement rappelé les conditions de l'analyse, nous justifierons le choix du type de plan d'expériences que nous avons utilisé, ainsi que la plage de variations définie pour chaque facteur. Les réponses que nous avons construites ont pour objectif de rendre compte des écarts par rapport à la valeur de consigne (au point de fonctionnement). L'exploitation utilise la technique de l'analyse de la variance et les calculs ont été menés à l'aide d'un logiciel de statistiques. Dans un dernier temps, nous essaierons de montrer comment il est possible de construire une fonction permettant de rendre compte de manière globale de la robustesse de l'analyse.

\section{Méthode d'analyse de la spiramycine}

L'analyse de la spiramycine dont la formule est donnée en figure 1 est actuellement obtenue par CPL Les mécanismes entrant en jeu lors de la séparation sont le partage à polarité de phases inversées et l'échange d'ions dynamique [6]. La méthode classique [32], telle qu'elle a été validée par Rhône-Poulenc Rorer, est la suivante : 


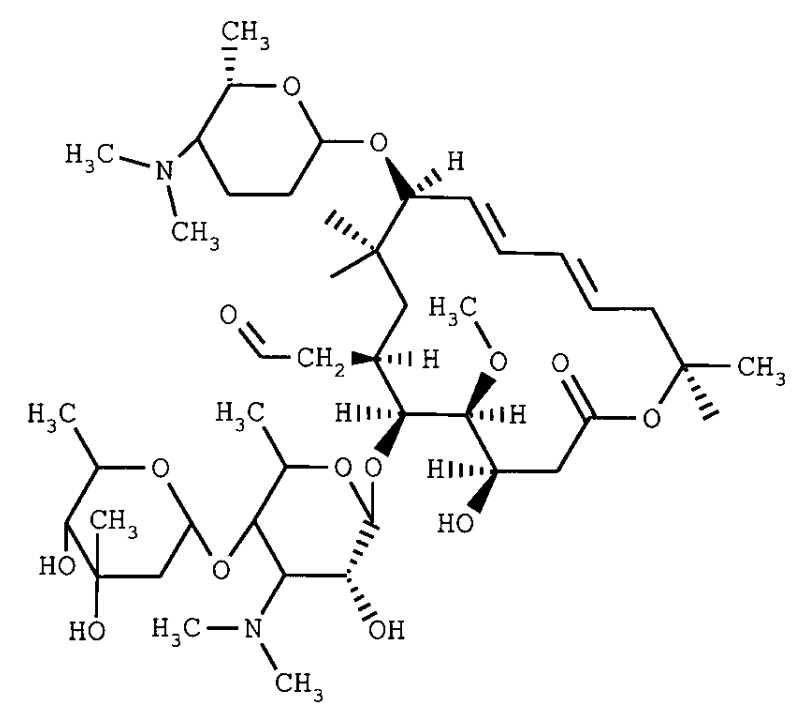

Fig. 1. Formule de la spiramycine I.

La colonne utilisée (Macherey-Nagel) est remplie avec une phase Nucléosil de silice greffée octyle. La granulométrie est de $3 \mu \mathrm{m}$ et la taille des pores $120 \AA$. Le diamètre interne de la colonne est de $4.6 \mathrm{~mm}$ et sa longueur de $20 \mathrm{~cm}$.

La phase mobile est un mélange de phase aqueuse et d'acétonitrile 70/30 v/v contenant $6.5 \mathrm{~g} \mathrm{l}^{-1}$ de perchlorate de sodium. L'acétonitrile doit être de qualité HPLC et ne pas contenir de cyanures. La phase aqueuse est obtenue en prélevant $6,7 \mathrm{ml}$ d'acide orthophosphorique (84\% minimum), $20 \mathrm{ml}$ de soude $0,1 \mathrm{M}$ et en complétant à 1 litre avec de l'eau de qualité HPLC. Le pH est ajusté à 2,2 avec de la soude $0,1 \mathrm{M}$.

L'analyse est réalisée en régime isocratique avec un débit fixé à $0,8 \mathrm{ml} / \mathrm{min}$. La colonne doit impérativement être thermorégulée à $23{ }^{\circ} \mathrm{C}$. La détection est assurée par un détecteur absorptiométrique UV réglé à $232 \mathrm{~nm}$. La quantification est menée à partir de l'aire des pics.

Les échantillons à analyser sont préparés de la manière suivante : on prélève par pesée exacte $50 \mathrm{mg}$ environ de spiramycine (poudre). On les dissout dans $20 \mathrm{ml}$ d'une solution eau/acétonitrile 70/30 v/v contenant du dinitro-3,4 toluène (DNT) à $1,5 \mathrm{~g} / \mathrm{l}$. On complète à $200 \mathrm{ml}$ avec un mélange eau/acétonitrile 70/30 v/v. Le volume injecté est de $20 \mu \mathrm{l}$. Le DNT joue uniquement le rôle d'étalon interne et est utilisé pour quantifier la spiramycine. Sitôt préparées, les solutions du principe actif doivent impérativement être conservées à $4{ }^{\circ} \mathrm{C}$.

Un exemple de chromatogramme est représenté sur la figure 2 .

\section{Plan d'expériences utilisé}

\section{Choix du type de plan}

Contrairement à ce qui peut être fait dans le cadre d'une optimisation de méthode, pour une étude de robustesse approfondie on ne peut se contenter d'un modèle polynomial de degré 1 (comme pourtant on le trouve fréquemment dans

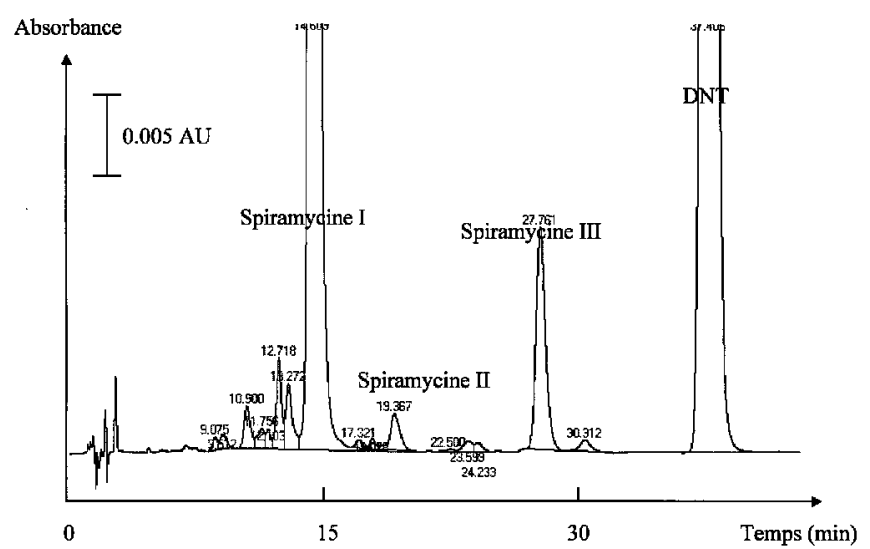

Fig. 2. Chromatogramme d'un échantillon de spiramycine, obtenu dans les conditions de la validation de la méthode (cf. texte pour conditions opératoires).

la littérature [7-19]) et donc de plans factoriels complets $2^{k}$ ou factoriels fractionnaires $2^{k-p}$. Il est nécessaire d'utiliser un modèle polynomial de degré au moins 2 [20], seul compatible avec la présence d'un extremum à l'intérieur du domaine expérimental. Cet extremum n'est autre que le point de fonctionnement et la méthode sera d'autant plus robuste que la courbure de la surface de réponse au voisinage de cet extremum sera faible.

Parmi les plans permettant l'utilisation d'un modèle polynomial de degré 2 nous avons choisi un plan composite centré $[21,22]$ qui présente des qualités optimales quant à la prévision de la réponse calculée en tout point du domaine. Le plan composite centré est en fait un plan factoriel de type $2^{k}$ (éventuellement factoriel fractionnaire $2^{k-p}$ ) auquel on a ajouté des points dits en étoile et des points au centre. Le nombre de points au centre ainsi que la distance des points axiaux au centre sont déterminés en fonction du critère d'isovariance par rotation (l'erreur sur le modèle de prédiction doit seulement être fonction de la distance au centre du plan) à l'aide du logiciel JMP [23].

Une visualisation de la répartition des points expérimentaux dans l'espace des facteurs pour un plan composite centré à trois facteurs est donnée en figure 3.

\section{Facteurs contrôlés et modèle}

Les expériences préliminaires ainsi que les connaissances déjà acquises sur la méthode nous ont conduit à choisir cinq facteurs. Ces facteurs sont ceux qui a priori sont les plus à même de jouer de manière significative sur les aires des pics et donc sur les titres. Ce sont pour la phase mobile : le débit, la concentration en ions perchlorate, le $\mathrm{pH}$ et le pourcentage volumique d'acétonitrile, et pour la colonne, la température. Le plan que nous avons décidé de retenir pour notre étude comporte 33 essais répartis de la manière suivante : 16 pour le plan factoriel fractionnaire $2^{5-1}, 10$ pour les points axiaux et 7 points au centre qui vont permettre d'estimer la répétabilité de la méthode et donc de tester la significativité des coefficients du modèle. Ce type de plan nous permet d'utiliser un modèle polynomial de degré 2 pour l'exploitation des résultats. L'équation du modèle postulé est la suivante : 


$$
\eta\left(X_{i, 1 \leq i \leq 5}\right)=\sum_{i=1}^{5} \beta_{i} X_{i}+\sum_{i=1}^{5} \beta_{i i} X_{i}^{2}+\sum_{i=2}^{5} \sum_{j=1}^{i-1} \beta_{i j} X_{i} X_{j}
$$

$\eta\left(X_{i, 1 \leq i \leq 5}\right)$ est la réponse théorique, elle est une fonction des cinq facteurs contrôlés $X_{i}(i=1$ à 5$)$. $\beta_{i}, \beta_{i i}, \beta_{i j}$ sont les coefficients du modèle.

La réponse expérimentale $Y\left(X_{i, 1 \leq i \leq 5}\right)$ est alors $Y\left(X_{i, 1 \leq i \leq 5}\right)=\eta\left(X_{i, 1 \leq i \leq 5}\right)+\varepsilon$ avec $\varepsilon:$ variable normale d'espérance mathématique nulle et de variance constante $\sigma^{2}$, qui correspond aux erreurs aléatoires de mesure. On recherche les estimations $b_{i}, b_{i i}, b_{i j}$ des coefficients du modèle mais on ne s'intéresse pas directement aux effets des facteurs.

Le plan fractionnaire utilisé a été construit en choisissant d'aliaser le cinquième facteur sur l'interaction des quatre autres, ce qui en notation de Box [24] s'écrit $5=1234$. Le générateur d'aliases de ce plan est donc $I=12345$.

Il apparaît alors que si l'on fait l'hypothèse que les interactions d'ordre 2 et plus sont négligeables, le plan choisi permet de calculer et de tester rigoureusement les coefficients des termes linéaires et quadratiques du modèle ainsi que tous ceux des interactions d'ordre 1 .

Le choix de la gamme de variations de chacun des facteurs est, dans l'optique d'une étude de robustesse, un problème délicat. En effet la plage de variations doit satisfaire deux critères qui peuvent se révéler contradictoires. D'une part la plage de variations des facteurs doit être suffisamment large pour que l'on puisse observer des variations significatives de la réponse et d'autre part elle doit être suffisamment restreinte pour pouvoir simuler les variations susceptibles de se produire de manière non contrôlée lors de la mise en œuvre de la méthode.

Pour un plan composite centré à 5 facteurs, le critère d'isovariance par rotation est vérifié lorsque la distance des

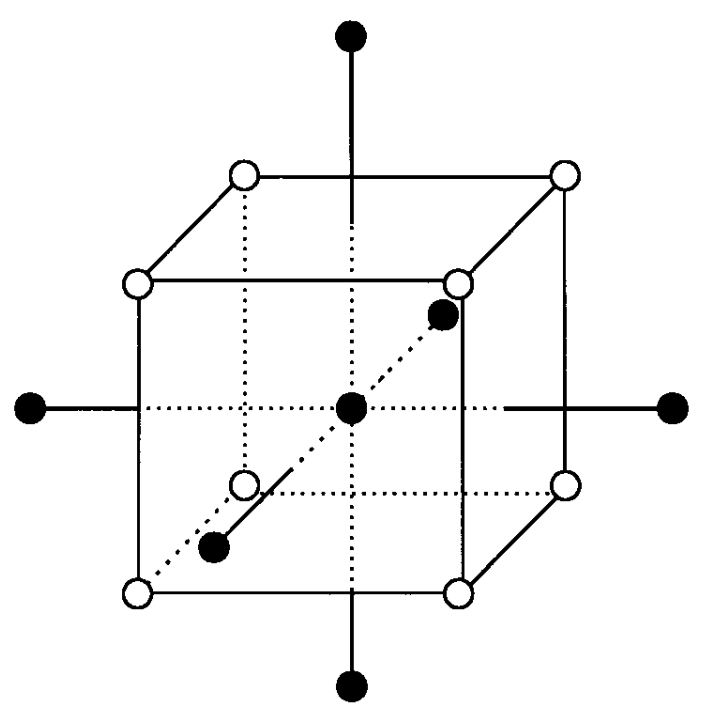

Fig. 3. Représentation dans un espace à trois facteurs d'un plan composite centré complet. En noir figurent les points en étoile et en blanc les points du plan factoriel. points axiaux au centre du domaine est égale à \pm 2 (en variables réduites).

L'ensemble de ces considérations nous a conduit a fixer les niveaux des facteurs comme indiqué dans le tableau I.

Tableau I. Niveaux des facteurs, les valeurs réduites correspondantes figurent entre parenthèses.

\begin{tabular}{lcccccc}
\hline & \multicolumn{3}{c}{ Niveau bas } & Point central & \multicolumn{2}{c}{ Niveau haut } \\
& $\begin{array}{c}\text { Axial } \\
(-2)\end{array}$ & $\begin{array}{c}\text { Factoriel } \\
(-1)\end{array}$ & $(0)$ & $\begin{array}{c}\text { Factoriel } \\
(+1)\end{array}$ & $\begin{array}{c}\text { Axial } \\
(+2)\end{array}$ \\
\hline Débit (ml/min.) & 0,76 & 0,78 & 0,80 & 0,82 & 0,84 \\
[perchlorate] $(\mathrm{g} / \mathrm{l})$ & 6,3 & 6,4 & 6,5 & 6,6 & 6,7 \\
$\mathrm{pH}$ & 2,0 & 2,1 & 2,2 & 2,3 & 2,4 \\
Température $\left({ }^{\circ} \mathrm{C}\right)$ & 21 & 22 & 23 & 24 & 25 \\
Acétonitrile $(\% \mathrm{v})$ & 28 & 29 & 30 & 31 & 32 \\
\hline
\end{tabular}

\section{Plan d'expérimentation}

L'ensemble des expériences réalisées au laboratoire est représenté dans le tableau II.

Tableau II. Structure du plan d'expériences.

Expérience $n^{\circ}$ Débit [Perchlorate] $p H$ Température \% Acétonitrile

\begin{tabular}{|c|c|c|c|c|c|}
\hline 1 & -1 & -1 & -1 & -1 & 1 \\
\hline 2 & -1 & -1 & -1 & 1 & -1 \\
\hline 3 & -1 & -1 & 1 & -1 & -1 \\
\hline 4 & -1 & -1 & 1 & 1 & 1 \\
\hline 5 & -1 & 1 & -1 & -1 & -1 \\
\hline 6 & -1 & 1 & -1 & 1 & 1 \\
\hline 7 & -1 & 1 & 1 & -1 & 1 \\
\hline 8 & -1 & 1 & 1 & 1 & -1 \\
\hline 9 & 1 & -1 & -1 & -1 & -1 \\
\hline 10 & 1 & -1 & -1 & 1 & 1 \\
\hline 11 & 1 & -1 & 1 & -1 & 1 \\
\hline 12 & 1 & -1 & 1 & 1 & -1 \\
\hline 13 & 1 & 1 & -1 & -1 & 1 \\
\hline 14 & 1 & 1 & -1 & 1 & -1 \\
\hline 15 & 1 & 1 & 1 & -1 & -1 \\
\hline 16 & 1 & 1 & 1 & 1 & 1 \\
\hline 17 & 0 & 0 & 0 & 0 & 0 \\
\hline 18 & 0 & 0 & 0 & 0 & 0 \\
\hline 19 & 0 & 0 & 0 & 0 & 0 \\
\hline 20 & 0 & 0 & 0 & 0 & 0 \\
\hline 21 & 0 & 0 & 0 & 0 & 0 \\
\hline 22 & 0 & 0 & 0 & 0 & 0 \\
\hline 23 & -2 & 0 & 0 & 0 & 0 \\
\hline 24 & 2 & 0 & 0 & 0 & 0 \\
\hline 25 & 0 & -2 & 0 & 0 & 0 \\
\hline 26 & 0 & 2 & 0 & 0 & 0 \\
\hline 27 & 0 & 0 & -2 & 0 & 0 \\
\hline 28 & 0 & 0 & 2 & 0 & 0 \\
\hline 29 & 0 & 0 & 0 & -2 & 0 \\
\hline 30 & 0 & 0 & 0 & 2 & 0 \\
\hline 31 & 0 & 0 & 0 & 0 & -2 \\
\hline 32 & 0 & 0 & 0 & 0 & 2 \\
\hline 33 & 0 & 0 & 0 & 0 & 0 \\
\hline
\end{tabular}


Les niveaux des facteurs sont exprimés en valeurs centrées réduites : 0 représente le niveau au point de fonctionnement (centre du domaine), -1 et +1 respectivement les niveaux bas et haut pour les points factoriels, -2 et +2 respectivement les niveaux bas et haut pour les points axiaux.

Il est clair que l'ordre indiqué dans le tableau II correspond à l'ordre de construction de la matrice des expériences et non à l'ordre dans lequel les essais ont été effectués.

\section{Réalisation expérimentale}

\section{Appareillage utilisé}

La chaîne chromatographique utilisée se compose d'une pompe Varian modèle 9012, d'un passeur-injecteur automatique réfrigéré Waters modèle 715 Ultra-Wisp, d'une jaquette de thermorégulation de la colonne à circulation d'eau alimentée par un bain thermostaté Bioblock de modèle Polystat 5, d'un détecteur absorptiométrique UV à longueur d'onde réglable de modèle Varian 2050 et d'une unité d'intégration composé d'un ordinateur compatible PC équipé du logiciel Class-VP de Shimadzu.

\section{Réalisation des essais}

La spiramycine présente une stabilité relativement limitée en solution aqueuse. Pour éviter le risque de voir les résultats de l'étude compromis par des problèmes de dégradation, nous avons décidé de limiter à quatre jours la durée maximale de stockage de nos échantillons en solution, et ce uniquement à condition que leur température de conservation soit de $4{ }^{\circ} \mathrm{C}$. Le nombre d'analyses à effectuer pour le plan nous a dissuadés d'utiliser une seule préparation. Afin de pouvoir utiliser deux préparations distinctes sans fausser les résultats nous avons eu recours à la technique de décomposition du plan en blocs. Le premier bloc se compose des points factoriels et de 6 points centraux et le second regroupe les point axiaux et un point central. Chacun des deux blocs d'expériences a été réalisé dans le laps de temps le plus court possible, sans interruption entre les expériences. Dans ces conditions, le fait d'utiliser deux préparations distinctes ne biaisera pas le calcul des estimations des coefficients du modèle. On respecte ainsi la limite de temps supérieure fixée pour la conservation des échantillons.

Afin de minimiser l'influence éventuelle d'une erreur systématique, les essais ont été réalisés dans un ordre aléatoire à l'intérieur de chaque bloc.

Grâce à la définition précise des conditions de chaque essai et du nombre d'essais à réaliser a priori l'utilisation d'un plan d'expériences nous a permis de planifier de manière précise le temps qu'il nous aura fallu allouer à notre étude. Cette possibilité qu'offrent les plans d'expériences prend une importance primordiale dès lors que l'on se place dans un contexte industriel.

\section{Protocole opératoire de chaque essai}

Chaque essai a été réalisé de manière aussi indépendante que possible des autres. Ainsi, un essai correspond à une préparation spécifique de phase mobile. Les phases mobiles ont été préparées de manière similaire à ce qui est décrit dans la méthode standard mais en prenant en compte les modifications à apporter aux niveaux de chaque facteur.

Ensuite pour chaque phase mobile la procédure a été identique. Afin d'assurer l'atteinte de l'équilibre, le temps imparti à la mise en équilibre entre les phases mobile et stationnaire est de deux heures, puis deux répétitions successives de l'analyse sont effectuées. On vérifie que le système est bien à l'équilibre grâce à la superposition des chromatogrammes. En tout, le plan représente 132 heures d'utilisation de la chaîne chromatographique.

Pour chacun des essais, chaque réponse chromatographique est obtenue en effectuant une moyenne des données recueillies sur les deux chromatogrammes.

\section{Construction des réponses}

\section{Réponses chromatographiques d'intérêt}

Dans le cas de l'analyse quantitative, ce sont les titres des différents composés du mélange, tout du moins les principaux, qui présentent le plus d'intérêt. Etant donné que les titres sont calculés directement à partir des aires, les réponses qui nous ont paru revêtir la plus grande importance sont les aires des produits normalisées par celle du DNT (pour être conforme à l'utilisation d'un étalon interne préconisé dans la méthode standard), la normalisation des aires des pics consistant à les rapporter à celle du DNT. Ensuite interviennent, au niveau de la reconnaissance des composés, les temps de rétention, puis de manière beaucoup plus secondaire des paramètres chromatographiques classiques tels la résolution, l'efficacité de la colonne ou l'asymétrie des pics. Cette démarche s'inscrit en marge de ce qui est fait habituellement où les études de robustesse sont uniquement menées sur un facteur de rétention ou une résolution $[7,11,12]$.

\section{Sélection des réponses}

Avant d'exploiter les résultats expérimentaux réponse par réponse il est judicieux d'effectuer une étude de corrélation entre les diverses réponses. En effet si deux ou plusieurs de celles-ci sont fortement corrélées cela signifie que leurs comportements vis-à-vis des facteurs envisagés dans le plan sont similaires et peuvent facilement se déduire les uns des autres par homothétie. Il apparaît donc inutile de multiplier des exploitations qui n'apporteront en fait aucune information supplémentaire. Nous verrons également par la suite comment utiliser cette étude de corrélation dans le cadre de la construction d'une fonction de robustesse.

\section{Pour illustrer ces propos prenons deux exemples :}

Le premier traite de la corrélation entre les différentes réponses chromatographiques liées au pic de spiramycine I. La figure 4 est une représentation des corrélations existant entre les différentes caractéristiques du pic de spiramycine I. Pour chaque couple de réponses, les valeurs obtenues lors des expériences sont représentées sur un graphe de type $X$, $Y$ ainsi que l'ellipse de confiance correspondante. Plus cette ellipse est proche d'un cercle plus la corrélation est faible, plus cette ellipse est aplatie plus la corrélation est forte.

Il apparaît clairement à l'examen de la figure 4 que les réponses ne sont pas corrélées entre elles. En pratique cela 


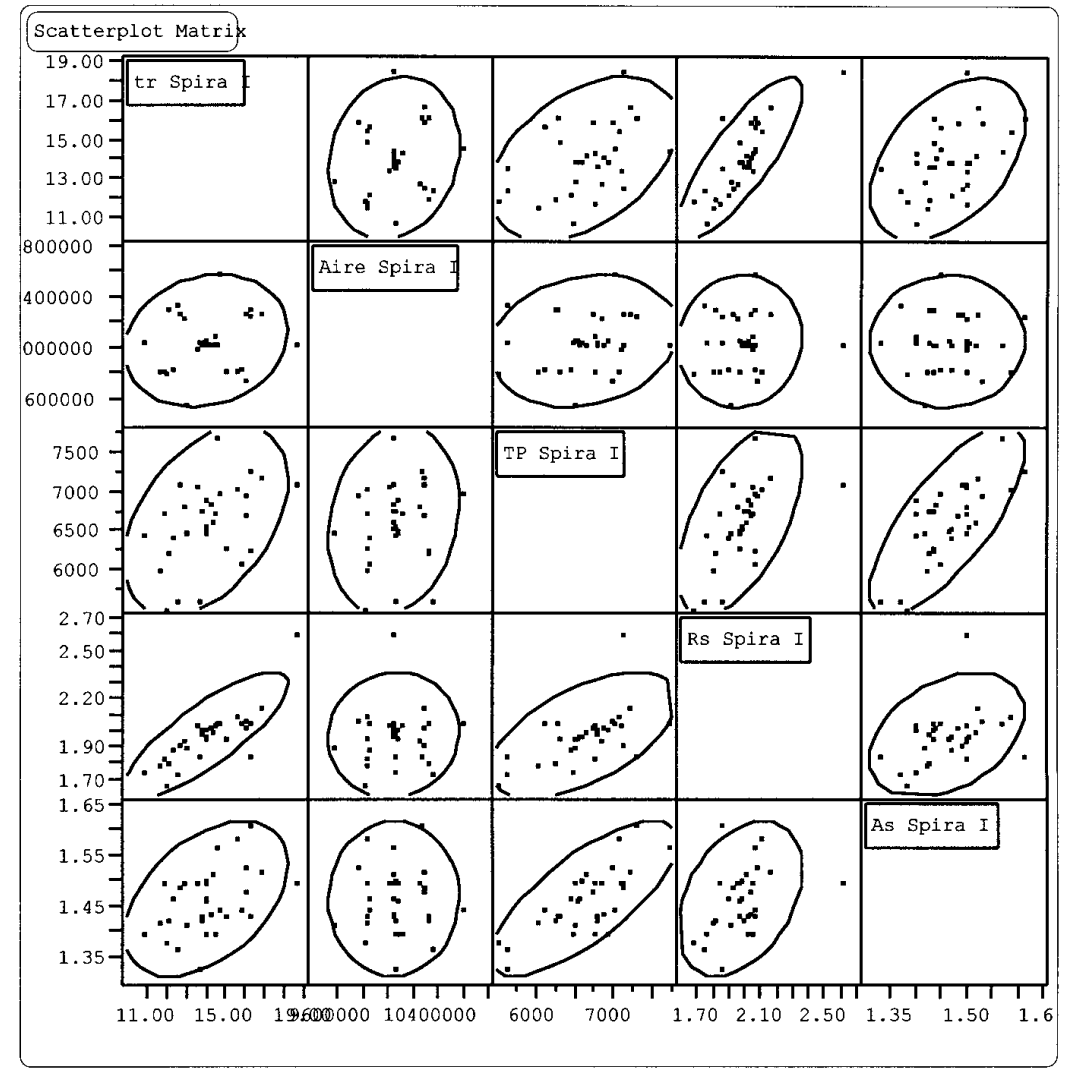

Fig. 4. Etude de corrélation entre les réponses chromatographiques liées à la spiramycine I ; les symboles tr, TP et As Spira I désignent respectivement le temps de rétention, le nombre de plateaux théoriques et l'asymétrie du pic de spiramycine I ; Rs Spira I désigne la résolution entre le pic de spiramicyne I et celui qui le précède immédiatement.

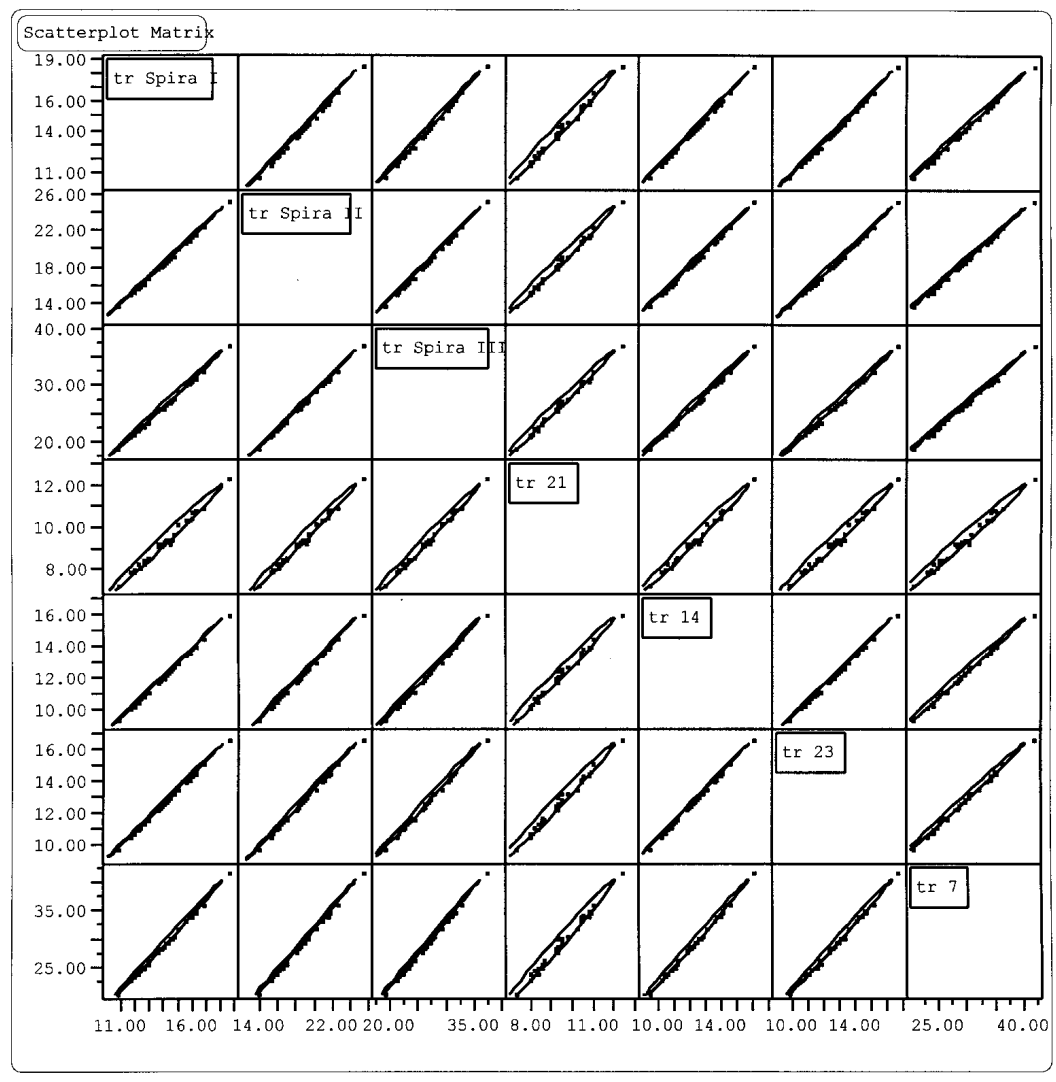

Fig. 5. Etude de corrélation entre les temps de rétention des spiramycines I, II et III et des principales substances apparentées. 
signifie que faire une exploitation pour chacune d'entre elles est profitable en termes de gain en information.

Le second traite de la corrélation entre les temps de rétention des spiramycines I, II, III et des principales substances apparentées. La figure 5 montre que les temps de rétention sont très fortement corrélés. En pratique cela signifie que faire une exploitation pour chacun n'apporterait rien en termes de gain en information. Il suffit de mener l'exploitation pour un seul : le temps de rétention de la spiramycine I.

Globalement les seules corrélations manifestes que nous ayons pu relever concernent les temps de rétention. Toutes les autres réponses peuvent être considérées comme indépendantes et feront l'objet d'une exploitation séparée. On peut noter que la forte corrélation entre les temps de rétention signifie que les facteurs choisis dans le plan ne peuvent en aucun cas permettre de jouer sur la sélectivité entre les composés, et du point de vue physico-chimique, que les trois spiramycines et les principales substances apparentées présentent des interactions similaires avec la phase stationnaire.

\section{Transformation mathématique}

Pour évaluer la robustesse d'un méthode d'analyse quantitative, la valeur de réponses brutes telles les temps de rétention ou les aires n'a directement aucun intérêt en soi. Ce qui doit retenir l'attention, ce sont les écarts relevés par rapport à une valeur de consigne (valeur mesurée au point de fonctionnement). D'autre part dans le cas d'une réponse destinée à estimer la robustesse, la valeur obtenue pour le point de fonctionnement doit être un extremum. Il nous semble plus parlant de choisir un maximum qu'un minimum.

Les consignes que nous nous sommes fixées pour la transformation mathématique des réponses brutes sont les suivantes :

- Valeur maximum pour la réponse moyenne $\bar{Y}$ au centre du plan (c'est-à-dire pour les valeurs de consigne des paramètres). Nous fixerons arbitrairement cette valeur maximum à 100 .

- Utilisation d'une relation polynomiale du second degré pour exprimer la réponse transformée $(Z)$ en fonction de la réponse brute $(Y)$.

- Possibilité de jouer sur l'«aplatissement» de la parabole en fonction des tolérances acceptées pour la réponse en question. Les tolérances, exprimées en $\%$, sont beaucoup plus larges par exemple sur les aires des impuretés que sur l'aire du produit principal, d'où l'utilisation de paraboles beaucoup plus aplaties pour les impuretés que pour le produit principal. Nous choisissons d'appeler $k$ le paramètre qui permet de jouer sur l'aplatissement de la parabole.

La transformation mathématique qui correspond le mieux à ces objectifs est une transformation de type parabolique (Éq. 2) dont une représentation graphique est donnée figure 6 .

$$
Z=\frac{100^{2}}{k Y^{2}} Y^{2}+2 \frac{100^{2}}{k Y} Y+100\left(1-\frac{100}{k}\right)
$$

Pratiquement, lorsque la réponse brute s'écarte de la valeur de consigne de $k \%$, la réponse transformée diminue
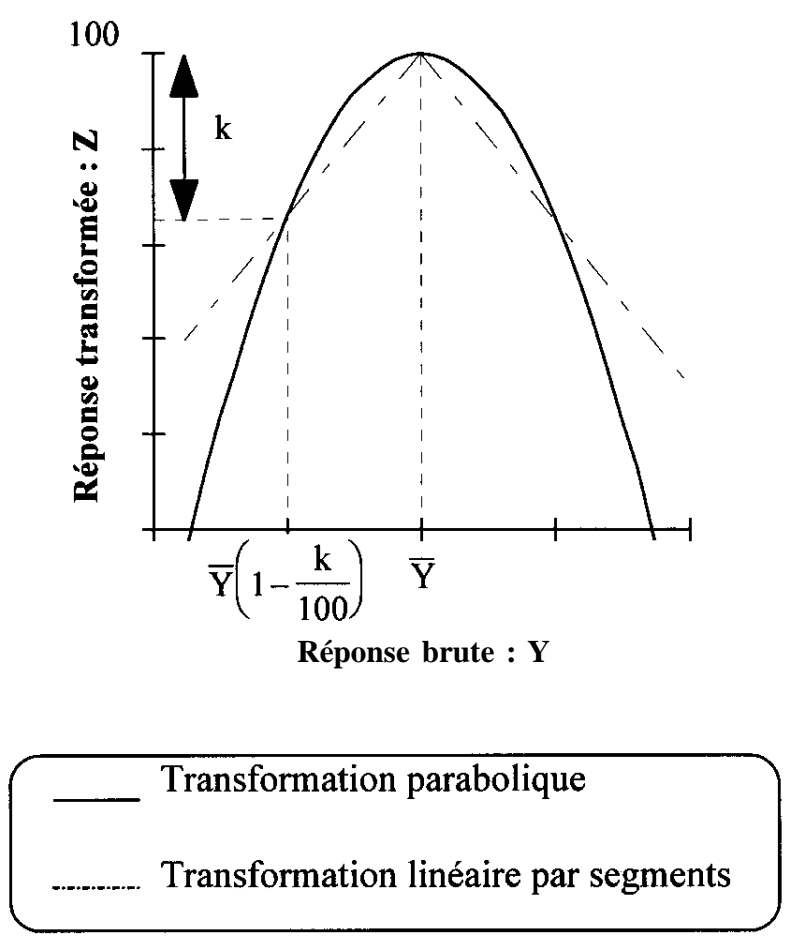

Fig. 6. Représentation graphique de la transformation parabolique appliquée.

de $k$ unités. Par rapport à une transformation linéaire par segments de type valeur absolue de l'écart à la valeur de consigne la transformation quadratique est plus «tolérante » tant que $Y$ est compris entre $\bar{Y}\left(1-\frac{k}{100}\right)$ et $\bar{Y}\left(1 \pm \frac{k}{100}\right)$ et plus « exigeante » sinon.

\section{Principe de l'exploitation}

Le principe de l'exploitation des résultats repose sur l'analyse de la variance [25]. Une analyse est effectuée pour chacune des réponses présentant un intérêt. Tous les calculs sont réalisés avec le logiciel JMP. Afin de mieux saisir concrètement les différentes étapes d'une exploitation nous allons utiliser un exemple comme support. Nous choisissons pour ce faire la transformée quadratique de l'aire normalisée du pic de la spiramycine III. Il est à noter que ce composé est considéré comme un produit principal et doit donc présenter de faibles marges de tolérance, d'où une valeur de $k$ fixée à 0,5 .

La première opération consiste à indiquer au logiciel le modèle que l'on désire utiliser, ici celui de l'équation (1).

Ensuite le logiciel estime la répétabilité grâce aux répétitions effectuées au point central et utilise cette estimation pour évaluer, à l'aide d'un test de Student [25], la significativité des coefficients calculés pour le modèle et considérés comme des variables aléatoires. En effet numériquement aucun coefficient du modèle ne sera jamais identiquement nul. Cependant statistiquement une valeur n'est jugée significativement différente de 0 que si la dispersion qui lui est associée ne permet pas d'expliquer la 
Tableau III. Coefficients du modèle pour la transformée quadratique de l'aire normalisée en spiramycine III.

\begin{tabular}{lccrrrr}
\hline Terme & Notation réduite & Coefficient & Estimation & écart-type & $t$ Ratio & Prob>|t|* \\
\hline Intercept & & & & & & \\
Débit & $I$ & $b_{0}$ & 98,59 & 0,56 & 177,21 & $<, 0001$ \\
ClO4- & $X_{1}$ & $b_{1}$ & 0,36 & 0,31 & 1,18 & 0,2598 \\
pH & $X_{2}$ & $b_{2}$ & 0,27 & 0,31 & 0,89 & 0,3910 \\
T & $X_{3}$ & $b_{3}$ & 0,98 & 0,31 & 3,18 & 0,0079 \\
ACN & $X_{4}$ & $b_{4}$ & $-1,57$ & 0,31 & $-5,11$ & 0,0003 \\
Débit*Débit & $X_{5}$ & $b_{5}$ & 0,59 & 0,31 & 1,93 & 0,0773 \\
ClO4-*ClO4- & $X_{1}^{2}$ & $b_{11}$ & 0,16 & 0,27 & 0,57 & 0,5769 \\
pH*pH & $X_{2}^{2}$ & $b_{22}$ & 0,06 & 0,27 & 0,21 & 0,8336 \\
T*T & $X_{3}^{2}$ & $b_{33}$ & $-0,16$ & 0,27 & $-0,59$ & 0,5685 \\
ACN*ACN & $X_{4}^{2}$ & $b_{44}$ & $-0,72$ & 0,27 & $-2,64$ & 0,0216 \\
Débit*ClO4- & $X_{5}^{2}$ & $b_{55}$ & 0,14 & 0,27 & 0,50 & 0,6246 \\
Débit*pH & $X_{1} * X_{2}$ & $b_{12}$ & $-0,34$ & 0,38 & $-0,91$ & 0,3828 \\
Débit*T & $X_{1}^{*} X_{3}$ & $b_{13}$ & $-0,12$ & 0,38 & $-0,32$ & 0,7540 \\
Débit*ACN & $X_{1}^{*} X_{4}$ & $b_{14}$ & 0,37 & 0,38 & 0,98 & 0,3481 \\
ClO4-*pH & $X_{1}^{*} X_{5}$ & $b_{15}$ & $-0,39$ & 0,38 & $-1,03$ & 0,3245 \\
ClO4-*T & $X_{2}^{*} X_{3}$ & $b_{23}$ & $-0,35$ & 0,38 & $-0,94$ & 0,3682 \\
ClO4-*ACN & $X_{2}^{*} X_{4}$ & $b_{24}$ & $-0,13$ & 0,38 & $-0,35$ & 0,7312 \\
pH*T & $X_{2}^{*} X_{5}$ & $b_{25}$ & $-0,17$ & 0,38 & $-0,45$ & 0,6626 \\
pH*ACN & $X_{3}^{*} X_{4}$ & $b_{34}$ & 1,11 & 0,38 & 2,97 & 0,0118 \\
T*ACN & $X_{3}^{*} X_{5}$ & $b_{35}$ & 0,08 & 0,38 & 0,21 & 0,8393 \\
\hline & $X_{4}^{*} X_{5}$ & $b_{45}$ & 0,54 & 0,38 & 1,43 & 0,1795 \\
\hline
\end{tabular}

* probabilité pour que la valeur calculée soit dépassée en module.

valeur observée et ce pour un risque d'erreur de première espèce $\alpha$ fixé.

Les résultats des calculs des coefficients du modèle pour la transformée quadratique de l'aire normalisée du pic de la spiramycine III sont reportés tableau III.

Il apparaît que si l'on fixe le risque de première espèce $\alpha$ à $5 \%$, peuvent seuls être considérés comme significativement différents de 0 les coefficients $b_{3}, b_{4}, b_{44}$ et $b_{34}$ qui correspondent respectivement aux influences des facteurs $\mathrm{pH}$, température, à l'influence quadratique du facteur température et à l'interaction $\mathrm{pH}$-température.

$\mathrm{Si}$ on tolère un risque $\alpha$ de $10 \%$, alors le coefficient $b_{5}$ peut également être considéré comme significativement différent de 0 . Ce terme correspond à l'influence du facteur « pourcentage volumique d'acétonitrile».

Il est possible de visualiser graphiquement les variations de la transformée quadratique de l'aire normalisée en spiramycine III en fonction des 2 facteurs les plus influents, à savoir le $\mathrm{pH}$ et la température (Fig. 7).

Cette visualisation permet, pour l'aire normalisée en spiramycine III, d'identifier la zone la plus affectée par des modifications des conditions expérimentales. En effet il apparaît clairement qu'il faut à tout prix éviter de se retrouver dans des conditions de température élevée et de $\mathrm{pH}$ faible puisque celles-ci conduisent à d'importantes modifications de la valeur de l'aire normalisée en spiramycine III.

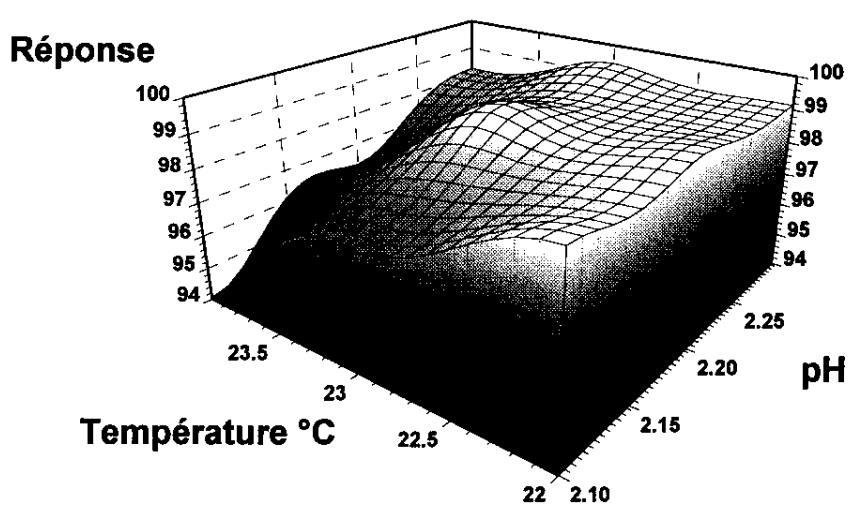

Fig. 7. Représentation graphique de la transformée quadratique de l'aire normalisée en spiramycine III en fonction du $\mathrm{pH}$ et de la température.

\section{La fonction de robustesse}

L'étude réalisée réponse par réponse est certes fort instructive et peut dans certains cas se révéler indispensable (spécifications très précises sur le titre d'un des produits) mais elle ne permet pas d'avoir une vision globale de la robustesse de la méthode. Les fonctions existant dans la littérature ne convenant pas à nos besoins [26-31], nous avons donc essayé de construire une fonction réponse de robustesse qui vise à reproduire de manière quantifiée et 
objective ce qui en général est laissé à l'appréciation intuitive de l'analyste expérimenté.

Le principe de construction de cette fonction de robustesse $F$ est simple puisqu'il consiste à combiner linéairement plusieurs fonctions réponses élémentaires $f_{i}$ comme indiqué dans l'équation (3).

$$
F=\sum_{i=1}^{n} c_{i} f_{i}
$$

De manière similaire à ce qui est décrit dans la littérature pour la construction des fonctions réponses chromatographiques utilisées en optimisation [6], le choix des fonctions élémentaires et des coefficients $c_{i}$ est laissé à l'analyste qui devra pour ce faire tenir compte du cahier des charges de la méthode d'analyse concernée et de l'étude de corrélation entre les réponses. Il est inutile de faire intervenir dans l'expression de la fonction réponse des réponses fortement corrélées entre elles. Dans notre cas nous avons choisi de prendre comme fonctions élémentaires les réponses suivantes :

- transformée quadratique du temps de rétention de la spiramycine I,

- transformées quadratiques des aires normalisées en spiramycine I, II et III,

- transformées quadratiques des aires normalisées des principales substances apparentées,

- résolution entre les pics de la substance apparentée 23 et de la spiramycine I,
- valeur absolue de l'écart à 1 des facteurs d'asymétrie des pics de spiramycine I et III,

- efficacité de la colonne mesurée sur les pics de spiramycine I et III.

La fonction de robustesse est construite de manière à fournir des valeurs d'autant plus élevées que la méthode est robuste.

Dans le tableau IV figurent les fonctions élémentaires utilisées. Les valeurs des coefficients $c_{i}$ découlent de la valeur de la contribution (poids que représente chaque terme $c_{i} f_{i}$ dans la fonction de robustesse) qui est fixée par l'analyste en fonction de ce à quoi il accorde le plus d'importance. Les contributions sont des données beaucoup plus informatives que les coefficients car ils permettent de comparer directement les poids des différentes fonctions élémentaires. Figurent également dans ce tableau les valeurs de $k$ utilisées en cas de transformation quadratique. Les résolutions, les valeurs absolues des écarts à 1 des asymétries et l'efficacité sont des fonctions pour lesquelles la transformation quadratique n'aurait aucun sens. En effet, pour ces termes, il n'existe pas de valeur de consigne mais par contre leur augmentation ne peut être que bénéfique pour la robustesse. Les valeurs des pondérations figurant dans le tableau IV correspondent à un choix particulier nous permettant d'illustrer notre méthodologie.

Les résultats de l'exploitation sur la fonction de robustesse sont rassemblés dans le tableau V.

Tableau IV. Coefficients et fonctions élémentaires intervenant dans l'expression de la fonction de robustesse.

\begin{tabular}{lccc}
\hline Fonction élémentaire $f_{i}$ & $k$ & Coefficient $c_{i}$ & Contribution \\
\hline $\begin{array}{l}\text { Transformée quadratique du temps de rétention } \\
\text { de la spiramycine I }\end{array}$ & 15,0 & 1,43 & $20 \%$ \\
$\begin{array}{l}\text { Transformée quadratique de l'aire normalisée } \\
\text { de la spiramycine I }\end{array}$ & 0,5 & 0,96 & $15 \%$ \\
$\begin{array}{l}\text { Transformée quadratique de l'aire normalisée } \\
\text { de la spiramycine II }\end{array}$ & 10,0 & 1,1 & $15 \%$ \\
$\begin{array}{l}\text { Transformée quadratique de l'aire normalisée } \\
\text { de la spiramycine III }\end{array}$ & 0,5 & 0,98 & $15 \%$ \\
$\begin{array}{l}\text { Transformée quadratique de l'aire normalisée } \\
\text { de la substance apparentée } 21\end{array}$ & 20,0 & 0,48 & $6 \%$ \\
$\begin{array}{l}\text { Transformée quadratique de l'aire normalisée } \\
\text { de la substance apparentée } 14\end{array}$ & 20,0 & 0,45 & $6 \%$ \\
$\begin{array}{l}\text { Transformée quadratique de l'aire normalisée } \\
\text { de la substance apparentée } 23\end{array}$ & 20,0 & 0,4 & $6 \%$ \\
$\begin{array}{l}\text { Transformée quadratique de l'aire normalisée } \\
\text { de la substance apparentée } 7\end{array}$ & 20,0 & 0,48 & $6 \%$ \\
$\begin{array}{l}\text { Résolution spiramycine I/substance apparentée } 23 \\
\text { Ecart à 1 des asymétries des pics de spiramycine I et III } \\
\text { Efficacité colonne }\end{array}$ & & 10 & $3 \%$ \\
\hline
\end{tabular}


Tableau V. Coefficients du modèle pour la fonction de robustesse.

\begin{tabular}{|c|c|c|c|c|c|c|}
\hline Terme & Notation réduite & Coefficient & Estimation & écart-type & $t$ Ratio & Prob $>|t|$ \\
\hline Intercept & $I$ & $b_{0}$ & 99,29 & 1,38 & 71,70 & $<, 0001$ \\
\hline Débit & $X_{1}$ & $b_{1}$ & 0,95 & 0,76 & 1,25 & 0,2355 \\
\hline $\mathrm{ClO} 4-$ & $X_{2}$ & $b_{2}$ & 1,31 & 0,76 & 1,72 & 0,1118 \\
\hline $\mathrm{pH}$ & $X_{3}$ & $b_{3}$ & $-1,76$ & 0,76 & $-2,31$ & 0,0398 \\
\hline $\mathrm{T}$ & $X_{4}$ & $b_{4}$ & $-1,79$ & 0,76 & $-2,34$ & 0,0371 \\
\hline $\mathrm{ACN}$ & $X_{5}$ & $b_{5}$ & 4,65 & 0,76 & 6,10 & $<, 0001$ \\
\hline Débit*Débit & $X_{1}^{2}$ & $b_{11}$ & $-0,08$ & 0,68 & $-0,12$ & 0,9080 \\
\hline $\mathrm{ClO} 4-* \mathrm{ClO} 4-$ & $X_{2}^{2}$ & $b_{22}$ & 0,07 & 0,68 & 0,10 & 0,9186 \\
\hline $\mathrm{pH}^{*} \mathrm{pH}$ & $X_{3}^{2}$ & $b_{33}$ & $-0,76$ & 0,68 & $-1,11$ & 0,2877 \\
\hline $\mathrm{T} * \mathrm{~T}$ & $X_{4}^{2}$ & $b_{44}$ & $-3,27$ & 0,68 & $-4,80$ & 0,0004 \\
\hline $\mathrm{ACN}^{*} \mathrm{ACN}$ & $X_{5}^{2}$ & $b_{55}$ & $-5,34$ & 0,68 & $-7,85$ & $<, 0001$ \\
\hline Débit*ClO4- & $X_{1} * X_{2}$ & $b_{12}$ & $-1,17$ & 0,93 & $-1,26$ & 0,2328 \\
\hline Débit*pH & $X_{1} * X_{3}$ & $b_{13}$ & $-0,39$ & 0,93 & $-0,42$ & 0,6814 \\
\hline Débit*T & $X_{1} * X_{4}$ & $b_{14}$ & $-0,96$ & 0,93 & $-1,03$ & 0,3236 \\
\hline Débit*ACN & $X_{1} * X_{5}$ & $b_{15}$ & $-1,85$ & 0,93 & $-1,98$ & 0,0716 \\
\hline ClO4-*pH & $X_{2} * X_{3}$ & $b_{23}$ & 1,35 & 0,93 & 1,45 & 0,1740 \\
\hline $\mathrm{ClO} 4-* \mathrm{~T}$ & $X_{2}^{*} X_{4}$ & $b_{24}$ & 1,15 & 0,93 & 1,23 & 0,2423 \\
\hline $\mathrm{ClO} 4-* \mathrm{ACN}$ & $X_{2} * X_{5}$ & $b_{25}$ & $-1,16$ & 0,93 & $-1,24$ & 0,2389 \\
\hline $\mathrm{pH}^{*} \mathrm{~T}$ & $X_{3}^{*} X_{4}$ & $b_{34}$ & $-0,61$ & 0,93 & $-0,65$ & 0,5266 \\
\hline $\mathrm{pH}^{*} \mathrm{ACN}$ & $X_{3} * X_{5}$ & $b_{35}$ & 0,81 & 0,93 & 0,86 & 0,4055 \\
\hline $\mathrm{T}^{*} \mathrm{ACN}$ & $X_{4} * X_{5}$ & $b_{45}$ & $-1,22$ & 0,93 & $-1,31$ & 0,2151 \\
\hline
\end{tabular}

D'après les calculs fournis par le logiciel il apparaît que si l'on fixe le risque de première espèce $\alpha$ à $5 \%$, peuvent être considérés comme significativement différents de 0 les coefficients $b_{3}, b_{4}, b_{5}, b_{44}$ et $b_{55}$ qui correspondent respectivement aux influences des facteurs $\mathrm{pH}$, température, pourcentage volumique d'acétonitrile, à l'influence quadratique des facteurs température et acétonitrile

Il est possible de visualiser graphiquement les variations de la fonction de robustesse en fonction de 2 facteurs, par exemple le pourcentage volumique d'acétonitrile et la température (Fig. 8).

La méthode utilisée pour l'analyse quantitative de la spiramycine réclame donc de la part de l'opérateur une attention toute particulière quant à la température de la colonne, au pourcentage d'acétonitrile et au $\mathrm{pH}$ de la phase mobile. La pérennité des résultats obtenus dépendra non seulement de la capacité à se placer aux valeurs de consigne mais également, et ce peut-être de manière encore plus critique, de la stabilité de ces facteurs au cours de l'analyse. Ainsi, comme le montre la figure 9, pour que la fonction réponse de robustesse ne soit jamais inférieure à 90 , il faut que la température soit maintenue au degré près et le pourcentage volumique d'acétonitrile au pour-cent près.

\section{Conclusion}

La méthode des plans d'expériences paraît bien adaptée à l'étude de la robustesse d'une analyse quantitative par CPL La principale difficulté réside en fait beaucoup plus dans la définition des objectifs, des facteurs, du domaine expérimental, dans le choix du type de plan, et dans la construction de réponses les mieux adaptées à une étude de

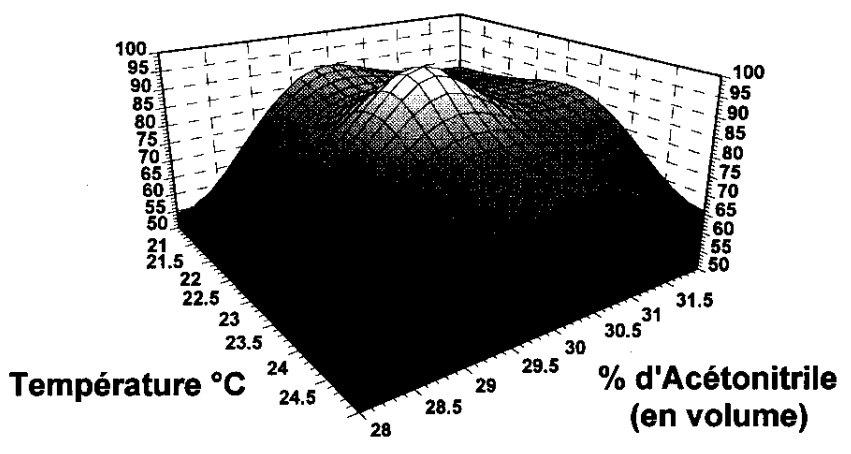

Fig. 8. Représentation graphique de la fonction de robustesse en fonction de la température et du pourcentage volumique d'acétonitrile.

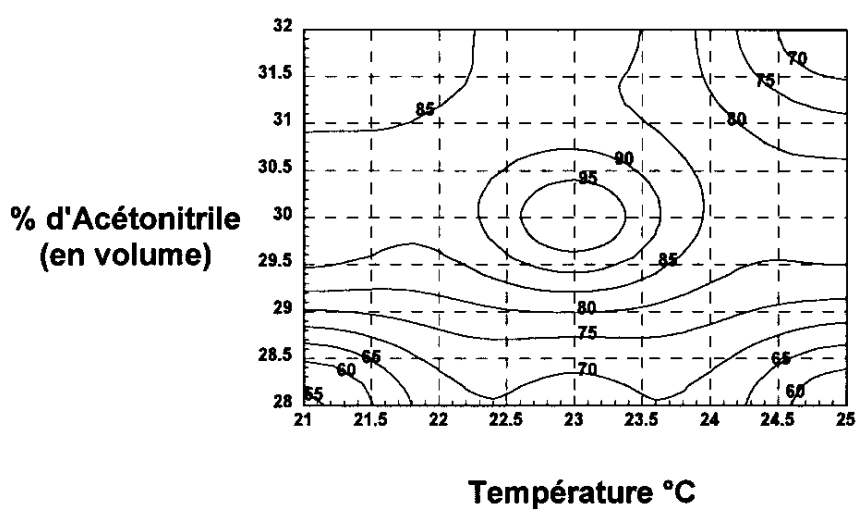

Fig. 9. Courbes d'isoréponses correspondant à la figure 8 . 
robustesse que dans les calculs numériques et l'interprétation des résultats. L'introduction d'une fonction de robustesse permet de caractériser globalement la capacité de la méthode à résister aux variations des conditions expérimentales, et d'identifier les facteurs qui requièrent la plus grande vigilance. Certes notre étude a été menée sur des aires normalisées et non directement sur des titres alors que la préoccupation essentielle de l'analyste en milieu industriel est l'insensibilité des titres produits à de petites variations involontaires des conditions opératoires. Or, la détermination des titres implique la mise en œuvre d'étalons externes pour l'estimation des coefficients de réponse. Du fait de l'augmentation de la durée des séquences d'analyse, l'étude directe de la robustesse menée sur les titres risquait de poser des problèmes liés à une possible dérive de l'appareillage d'une part, à la stabilité limitée des échantillons d'autre part. Dans un second temps, nous poursuivrons cette démarche expérimentale par une étude approfondie sur les titres prenant en compte uniquement les trois facteurs influents déterminés au cours de la présente étude, afin de vérifier directement la robustesse de la méthode sur les titres.

\section{Remerciements}

Nous tenons à remercier tout particulièrement MM. A. Brun, P. Anger, J.M. Menet et L. Burbaud de Rhône-Poulenc Rorer, Chemical and Pharmaceutical Development, pour leur aide et leur soutien.

\section{Références}

1. Jenke, D. R. J. Liq. Chrom. Rel. Technol. 1996, 19(12), 18731891.

2. Goupy, J. In : La méthode des plans d'expérience, Dunod, Paris, 1988.

3. Jones, K. Int. Lab. 1986, 16(6), 32-45.

4. Mulholland, M.; Waterhouse, J. Chromatographia 1988, 25(9), 769-774.

5. Mulholland, M.; Waterhouse, J. Chem. Intell. Lab. Syst. 1989, 5, 263-270.

6. Rosset, R.; Caude, M.; Jardy, A. In : Chromatographies en phases liquide et supercritique, Masson, Paris, 1991.

7. Vander Heyden, Y.; Hartmann, C.; Massart, D. L.; Michel, L.; Kiechle, P.; Erni, F. Anal. Chim. Acta 1995, 316, 15-26.

8. Chaminade, P.; Feraud, S.; Baillet, A.; Ferrier, D. S.T.P. Pharma Pratiques 1995, 5(1), 17-35.

9. Virlichie, J. L.; Ayache, A. S.T.P. Pharma Pratiques 1995, 5(1), 49-60.

10. Virlichie, J. L.; Ayache, A. S.T.P. Pharma Pratiques 1995, 5(1), 37-48.

11. Vander Heyden, Y.; Luypaert, K.; Hartmann, C.; Massart, D. L.; Hoogmartens, J.; De Boer, J. Anal. Chim. Acta 1995, 312, 245-262.
12. Massart, D. L.; Perelgritz, L. Analusis 1994, 22(5), M14-M37.

13. Caporal-Gauthier, J.; Nivet, J. M.; Algranti, P.; Guilloteau, M.; Histe, M.; Lallier, M.; N'Guyen-Huu, J. J.; Russotto, R. S.T.P. Pharma Pratiques 1992, 2(4), 205-226.

14. Caporal-Gauthier, J.; Nivet, J. M.; Algranti, P.; Guilloteau, M.; Histe, M.; Lallier, M.; N'Guyen-Huu, J. J.; Russotto, R. S.T.P. Pharma Pratiques 1992, 2(4), 227-239.

15. Leeuwen, J. A.; Vandeginste, B. G. M.; Kateman, G.; Mulholland, M.; Cleland, A. Anal. Chim. Acta 1990, 228, 145153.

16. Leeuwen, J. A.; Buydens, L. M. C.; Vandeginste, B. G. M.; Kateman, G.; Mulholland, M. Anal. Chim. Acta 1990, 235, $27-$ 40.

17. Leeuwen, J. A.; Buydens, L. M. C.; Vandeginste, B. G. M.; Kateman, G.; Schoenmakers, P. J.; Mulholland, M. Chemom. Intell. Lab. Syst. 1991, 10, 337-347.

18. Leeuwen, J. A.; Buydens, L. M. C.; Vandeginste, B. G. M.; Kateman, G.; Schoenmakers, P. J.; Mulholland, M. Chemom. Intell. Lab. Syst. 1991, 11, 37-55.

19. Leeuwen, J. A.; Buydens L. M. C.; Vandeginste, B. G. M.; Kateman, G.; Cleland, A.; Mulholland, M.; Jansen, C.; Maris, F. A.; Hoogkamer, P. H.; van den Berg, J. H. M. Chem. Intell. Lab. Syst. 1991, 11, 167-174.

20. Bourguignon, B.; Marcenac, F.; Keller, H. R.; de Aguiar, P. F.; Massart, D. L. J. Chromatogr. 1993, 628, 171-189.

21. Mulholland, M. Trends Anal. Chem. 1988, 7(10), 383-389.

22. Hendriks; M. M. W. B.; De Boer, J. H.; Smilde, A. K. In: Robustness of analytical chemical methods and pharmaceuti cal products, Data Handling in Science and Technology, Vol. 19; Elsevier, Amsterdam, 1996.

23. SAS Institute, JMP Statistic and Graphics Guide and User's guide (Version 3.1), USA, 1995.

24. Box, G. E. P.; Hunter, W. G.; Hunter, J. S. In: Statistics for Experiments, An Introduction to Design, Data Analysis and Model Building; Wiley, New York, 1978.

25. Commissariat à l'Energie Atomique, In : Statistique appliquée à l'exploitation des mesures, I and II; Masson, Paris, 1978.

26. De Boer, J. H.; Smilde, A. K.; Doornbos, D. A. Chem. Intell. Lab. Syst. 1990, 7, 223-236.

27. De Boer, J. H.; Smilde, A. K.; Doornbos, D. A. Chem. Intell. Lab. Syst. 1991, 10, 325-336.

28. De Boer, J. H.; Smilde, A. K.; Doornbos, D. A. Chem. Intell. Lab. Syst. 1991, 15, 13-28.

29. Vanbel, P. F.; Tilquin, B. L.; Schoenmakers, P. J. J. Chromatogr. A 1995, 697, 3-16.

30. Bourguignon, B.; Massart, D. L. J. Chromatogr. 1991, 586, 1120.

31. Smilde, A. K.; Knevelman, A.; Coenegracht, P. M. J. J. Chromatogr. 1986, 369, 1-10.

32. Jardy, A.; Vial, J.; Ménier, I. Analusis 1997, 25, 106-111. 